\title{
Systemic Model of Organizations
}

\author{
Gustavo de Jesús Pérez Durán \\ Universidad Autónoma de Guadalajara, Guadalajara, Jalisco, Mexico \\ Email: Gustavo.perez.g@gmail.com
}

How to cite this paper: de Jesús Pérez Durán, G. (2021). Systemic Model of Organizations. Open Journal of Business and Management, 9 , 1230-1245.

https://doi.org/10.4236/ojbm.2021.93066

Received: March 15, 2021

Accepted: May 25, 2021

Published: May 28, 2021

Copyright (c) 2021 by author(s) and Scientific Research Publishing Inc. This work is licensed under the Creative Commons Attribution International License (CC BY 4.0).

http://creativecommons.org/licenses/by/4.0/

\begin{abstract}
A new definition of system is used, which is the foundation of a new theory called Specific Theory of Systems and based on which a hypothesis is deduced about which is the systems-based model that reliably describes how organizations are integrated and how do they work. This model is described in detail and is proposed as a hypothesis to serve as a basis for a subsequent empirical investigation that allows it to be verified for its acceptance or rejection. The purpose of the research was to use a new system theory called STS, developed by the author in previous works, to deduce in a logical way, a structure model that allows describing organizations based on systems, in this case, socio-technical systems. The method used was to deduce from the concepts of the STS, how the structure that integrates and interrelates the systems that make up an organization should be formed. The conclusion obtained was that the fundamental system of the structure is the one in which a result or final product of the organization is assembled and that this system is fed by a set of systems that provide inputs and another set that provides general services. In addition, each of the feeder systems also generates a similar structure and each of their feeder systems also does so in such a way that the structure can be developed by levels until reaching an adequate, required level or until reaching the limit of the organization that it is studied.
\end{abstract}

\section{Keywords}

System, Organization, Process, Structure, Model

\section{Introduction}

This work presents the results of an investigation based on a new systems theory developed by Pérez (2020b), Pérez (2020a) and Pérez (2020c) which has been called the Specific Theory of Systems (STS), since it allows identifying what the systems are and differentiating them from other entities that are not. Based on this theory, it is clearly deduced which is the system configuration that correctly 
describes any organization and thus provides a solid base to help in managing them.

In the existing literature, one can find various attempts made to postulate a system configuration as a basis to describe what an organization is and how it works, but in all cases, the General Theory of Systems (TGS) proposed by Bertalanffy (1968), has been taken as a basis, implicitly or explicitly, and the interpretations and derived models have been, in some cases of very little practical utility and in others, too vague descriptions of the configuration that coherently integrates the systems to support the functioning of organizations.

Here, a systemic model is proposed that accurately describes the configuration on which systems are integrated in any organization and that will serve as the basis for developing more precise and practical management approaches and will surely also serve to design better organizational schemes and structures.

The research presented in this article is a qualitative research developed in the deductive stage. The logical steps that were taken to deduce from a new basic theory (STS), the configuration of systems that accurately describes what an organization is and how it works, are analyzed in detail. What is presented here is a hypothesis about such a configuration, which must be contrasted with reality in order to accept or reject it.

To start the discussion, the fundamental concepts of the new system theory on which the research is developed, which has been called STS, are presented. The four best-known models in the literature on the interpretation of an organization as a structure made up of systems and that have been used to develop management models, business software, management strategies and business analysis methods, among other things, are reviewed below. Finally, the systemic structure of an organization is deduced based on the fundamental concepts of STS theory.

\section{Background}

In three previous publications, Pérez (2020b), Pérez (2020a) and Pérez (2020c) the author analyzed the defects that the definition of a system originally proposed by Bertalanffy (1968) has. It has been used by all researchers up to the present, with some additions and clarifications that do not modify its original meaning. Adams et al., (2014) proposed a definition of Systems Theory, which can be applied to systems themselves, but uses as a basis for their generalization-oriented inferences, properties and behaviors observed in systems, but which they are not generalizable and therefore this definition is not usable as a basis for the concept of system. Furthermore, the behaviors of an entity, whatever it may be, describe only its external effects, but do not describe its essence, which is what a definition must explain.

Bertalanffy developed his concept of systems by studying existing systems in living beings, but perhaps in an attempt to ensure that it could be applied in a generalized way to many other situations, he selected, as a basis for the definition he proposed, only generic aspects that had no relation with the functioning 
of the systems to ensure that they could be identified in other fields of science and reality. Bertalanffy defined a system as "a complex of interacting components" (Bertalanffy, 1968: p. 56), never specifying what a component is, or what interacting is. If this definition is analyzed, it is immediately detected that, according to Bertalanffy, a system is a set of things, of any kind, that act with each other or interact or are related in some way, whatever this may be. But if you reflect a bit, you can easily come to the conclusion that all things existing in our reality are made up of elements or components that are linked or interrelated in some way.

In another way, Bertalanffy established that anything or object existing in nature is a system: a stone, a river, an organism, and whatever else exists in reality, is a system. So it is possible to say that Bertalanffy's definition does not it defines neither a system nor anything specific. A definition should describe the essential characteristics that can be identified only in certain types of entities, which when possessed them immediately are considered part of the different and exclusive set of entities that satisfy said definition. The definition makes it possible to identify the entities that meet the characteristics contained in it and exclude those that do not meet them. In other words, the definition must allow identification and discrimination. Bertalanffy's definition of what a system is does not allow discrimination, it accepts anything as a system.

The definition of a system used up to now is so trivial that many researchers carry out studies, dissertations and research, without thinking about the definition of a system. In many scientific articles, laws and concepts about systems are discussed, analyzed and proposed without having established, before and clearly, what a system is. The meaning of the word system is left to the interpretation of the reader and the properties or behaviors that they have in certain specific fields of reality are discussed freely. In accordance with the generality of the definition of a system accepted and used extensively, when speaking of systems, one speaks of anything that exists and the question that arises is: do all existing things have properties or behaviors such as those established by Bertalanffy (1968) for living systems? For example, properties like equifinality and homeostasis? Obviously not, and yet many authors propose it, even if they have to give different interpretations to these concepts.

According to Pérez (2020c), a system is a totality that meets the following six characteristics:

1) Components. Systems are made up of a set of components that perform consistent and determined actions.

2) Structure. The systems have a structure made up of interconnections between the components that allow the exchange of matter, energy or information.

3) Organization. The systems have an organization that establishes the actions that each component must perform.

4) Process. The systems work based on a process that establishes the way to combine the actions of the components in order to obtain the final expected re- 
sult of the system, which can be actions or materials. The processes can receive inputs that are transformed into material products of the system and if they do not receive them, then the results of the system are actions.

5) Products. They are the results obtained from the process, which can be actions or materials, in order to be material, the process must have the capacity to receive material inputs that are transformed through actions carried out by the components.

6) Energy. The systems use energy to generate the actions performed by the components, which can be received from the external environment or generated by the system based on materials also obtained from the external environment.

A system must have each and every one of these characteristics, it will be enough that it lacks one of them so that the corresponding entity is not considered a system. This way of defining what a system is, meets the two fundamental requirements of a definition: it accurately describes what a system is and makes it possible to determine whether an entity is a system and at the same time discriminate those that are not, therefore both: delimits and discriminates.

There are three basic types of systems:

a) Natural systems. Those created by nature, among which are predominantly living systems.

b) Electromechanical, chemical, nuclear, physicochemical and any other system created by human beings based on the technology developed, predominantly computers.

c) Social systems, also created by human beings, but whose main characteristic is that the components of the system are, at the same time, human beings.

The definition of a system that was established in a previous paragraph, is generally applicable to the three types of systems, all of them show the six characteristics that describe and distinguish a system. This definition is then general enough to cover all systems and specific enough to determine what a system is not.

\subsection{Katz and Kahn}

Katz and Kahn (1966) propose a model to understand and analyze organizations, which is based on the open system concept managed by Bertalanffy (1968). According to these authors, an organization is an open system that receives materials and energy from the outside and generates products that are consumed by the outside and with this it is possible to obtain new flows of materials and supplies that keep the system in constant operation. They also establish that this open system is made up of five subsystems that have different but complementary purposes, these subsystems are:

1) Production: primary processes. Task fulfillment and energy transformation.

2) Maintenance of the work structure. Mediation between task demand and human needs to maintain the operating structure.

3) Border systems. 
a) Production support: procurement of materials and labor and disposal of products. Exchange transactions at the borders of the system.

b) Institutional system. Obtaining social support and legitimation.

4) Adaptation. Intelligence, planning, research and development.

5) Managerial management.

a) Resolution of conflicts between hierarchical levels.

b) Coordination and direction of functional substructures.

c) Coordination of external requirements and resources and needs of the organization.

Katz and Khan freely use the broad ambiguity that exists in the definition of a system that they adopted from the General Theory of Systems (TGS) and therefore postulate that an organization is an open system, that is: some set of elements that are related and interact. However, they never try to explain or describe those elements or components, they simply say that the organization is a system whatever it is.

To further complicate matters, Katz and Khan postulate that the system called organization is made up of five subsystems that are not described with breadth and clarity either and that are as complex as the total system to which they belong. By using such a general definition of what a system is, these authors propose as systems large conglomerates of people and activities that are only identified as parts of a whole through the name of some generic function such as: production, planning, procurement, etc.

What Katz and Khan are really describing as subsystems of the system called organization, are large complex functions that are performed in the organization and that involve people from different specialties and functional areas, but that can hardly be considered systems. Given the breadth and complexity of these great functions, it is impossible to identify the components and the other five characteristics that allow a system to be recognized. Perhaps they are large conglomerates of systems that act in a coordinated way. What Katz and Khan call subsystems, do not include, for example, a specific process integrated by actions through which the final results are obtained. They are then intellectual creations generated based on inferences and interpretations of these authors, but not systems in the strict sense of their definition.

It can then be said that the analysis carried out by Katz and Khan manages to describe an organization based on certain generic functions that facilitate the understanding of an extremely complex entity and allow its study and management in an orderly and logical way, but they could have done so without using the concept of system.

\subsection{Socio-Technical Systems}

Trist (1981) reports that the concept of socio-technical systems began in 1949 as part of various field projects carried out by the Tavistoc Institute in the British coal mining industry. Trist describes that a work system is made up of a tech- 
nological system made up of machines that function as tools to help humans perform some actions and by a social system made up of people. The approach described by Trist, considers these two systems as different entities, but studies them under the principle that they cannot be optimized separately, since if this were done, the independent optimization of only one of them implies that their joint operation will not be optimal.

Trist establishes that they followed a systems-based approach, since the concept of socio-technical systems was developed based on interdependencies and that the concept of open systems was used as another foundation, since it was considered that the organization has to maintain a state of equilibrium with its external environment. It also points out that both the field work and the theoretical construction were influenced by (Bertalanffy, 1950).

This systemic approach was complemented, in the social part, with some innovations in relation to the way of managing the organization. The formation of groups was consolidated in order to promote the identification of work units instead of considering only individual work, internal regulation of the system by the group was promoted instead of external regulation of individuals through supervisors and the generation of skills and multiple capacities in the workers was managed to increase the repertoire of responses from the group.

The first thing that can be observed in the work carried out by the Tavistoc Institute researchers is that they adopted the systems concepts developed by Bertalanffy and that the only defining property they used for a system was that they were handling interdependent elements. In other words, they considered that their two systems: the social system and the technological system, are systems because they are made up of interrelated components, which is just one of the characteristics of a system, according to the definition that was given at the beginning of this writing, and it may not be the most important nor the one that best helps to define a system. It must be remembered that the quality of being made up of interrelated components is a characteristic that all things existing in our world or in the universe have. Therefore, there are very few properties or functionalities that could be obtained from that consideration.

What is observable in the socio-technical systems is that they are specifically located in the production line of the coal mines, they are part of the process that allows the extraction of coal. This means that the Tavistoc Institute researchers were studying a process and not two separate systems as they raised it, however it turns out that it was an effort made on the process although that was not their main intention.

In studying a process, these researchers were actually studying a system although they only fixed their attention on the social and technical parts of it and never observed it in its entirety. In any case, socio-technical system is an effort aimed at interpreting an organization as something integrated by systems, it is perhaps one of the first serious efforts in that sense, despite the fact that it fell short in the systemic vision. 


\subsection{Quality Control}

In 1930, WA Shewhart published an article on statistical quality control in the manufacture of telephone equipment, in which it established that the manufacturing process of telephone sets is like a funnel and in its final section of small dimensions, $100 \%$ of the manufactured equipment is inspected in order to ensure its quality and protect customers. Shewhart (1930) states that it is more economical to discard defective materials in the initial stages of production, than to allow them to continue until the final stage where they will probably cause the rejection of a finished product. In summary, Shewhart states that controlling the quality of the process in all its stages ensures the quality of the product.

Shewhart, directs his attention to the process and not to the final product, with these studies the approach that today is called "Statistical Process Control" begins. It is the beginning of the trend to manage processes to ensure that its various stages work well to ensure that the final product is correct. Shewhart is really studying the manufacturing system but he does not look at it as a system, he only focuses on the process that is one of the elements that make up the system. It is important to note that Shewhart does not analyze the company as a whole, he only focuses on the manufacturing system of the final product, but with this, the interest in applying the concept of system in business management begins.

Feigenbaum (1951) raises a new concept of quality control, postulates that quality control must involve all phases of the industrial production process, according to Feigenbaum, it must start with the customer's specifications, and continue in several other stages: engineering, laboratory, procurement, manufacturing methods, work planning, inspection and testing, packaging, distribution and return to customers, all in a continuous cycle of quality control.

It can be seen that Feigenbaum no longer concentrates on the manufacturing process, it proposes to manage other processes and integrate them all in a cycle of permanent improvement and where the needs of all of them are met. For this reason, Feigenbaum is considered the pioneer of the concept of Total Quality. This author continues to observe the manufacturing process but does not conceive it as a system and adds other areas of the company but describes them as specialties or conglomerates of activities and does not interpret them as systems, considers the value of their results, but does not identify in them some systemic characteristic. Obviously, when considering that all those specialties involved in continuous cycles of change and adjustment are processes that generate results, he is somehow adopting the concept of a system, since all processes are part of some system.

Ishikawa (1986) reports that in 1950 Dr. Edward Deming gave various lectures on quality control methods and this meant the beginning of a movement that radically changed the work methods and the administrative approach. The most important contribution of the Japanese people was to bring quality control to the entire organization. They considered that everyone should work under the mod- 
el of continuous quality improvement and not only on the production lines. Its methodology was always based on processes: the sets of activities that allow obtaining a result and also considered that the results are the consequence of processes that are concatenated.

Again, when considering processes, the Japanese were managing systems, even if they were not explicit, and their approach was to conceive of the company as a complex network of linked systems. However, they did not propose any model that could be used to describe their network of systems and give meaning and structure to their systemic conception.

\section{Horizontal Processes}

Ostroff (1999) proposed a model to analyze and manage an organization. Ostroff argues that there are fundamental processes that allow delivering the company's value proposition to customers. These processes are constituted by different sets of activities dispersed in several of the vertical functions of the organization and the horizontal approach that Ostroff proposes, seeks to integrate all these groups in a single great process, to manage and operate it as a single administrative unit. The organization requires a few fundamental processes and the model to analyze and manage it is, in general, as shown in Figure 1.

It is observed that they are large processes that are horizontal because they cross the organization as they are integrated by sets of activities of all or several vertical functions. Ostroff uses a very general concept of process, he implicitly considers that a process is a set of activities of any kind, simple, complex, specific or general, whatever one can imagine. Obviously they are interrelated activities and hence they really represent large systems, according to the traditional concept of TGS. So the Ostroff Horizontal Organization is, basically, a way of considering the organization integrated by systems.

Rummler and Brache (2013) used a model in which the company is interpreted through systems, although they did not declare the concept of the system they used or gave a definition of system. They postulate that an organization is a processing system that converts inputs into outputs or products that are

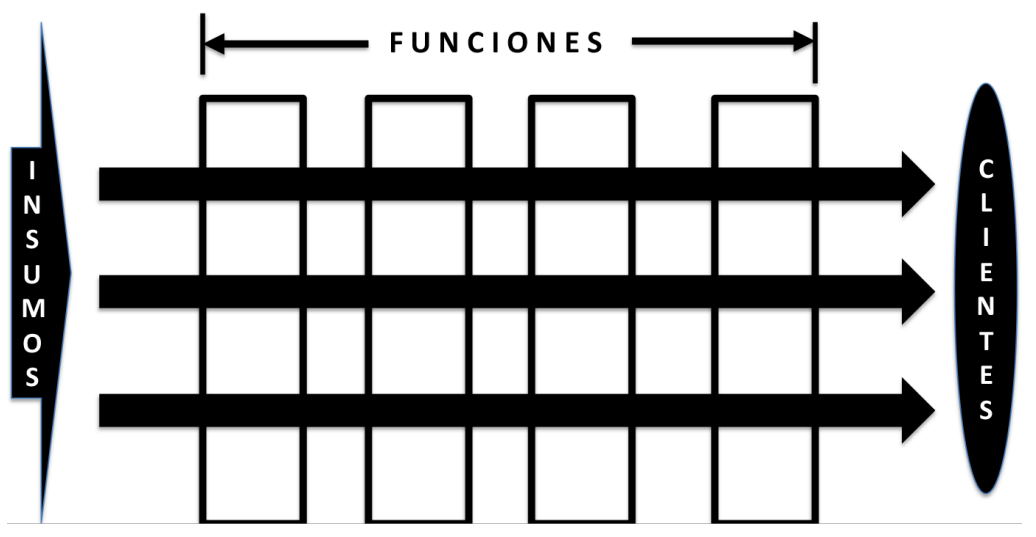

Figure 1. Horizontal model of processes. 
delivered to other systems that are receivers.

They analyze the organization on three levels, the first in which it is seen as a complete system and only its relations with the market and with its shareholders are considered. In the second plane in which they show how the organization works and the model they use to illustrate it is exactly the horizontal process model shown in Figure 1. Finally, at the third level they establish that the processes are executed and managed by people who carry out various jobs.

The important thing is that in their management approach they consider that the organization works based on horizontal processes that go through and integrate various sets of activities belonging to different functions. All this is used to develop an analysis and management methodology that has been widely used in the design of methodologies and tools for the development of management support computer systems.

The concept of horizontal processes that end directly with customers was adopted to develop a process-based management approach, which is described by Benedict et al. (2013). The model they use in their methodology is exactly the one shown in Figure 1, but they specify that the important processes are those that generate value for customers. These authors based their book on a concept that was already in use by some consulting firms and which was called BPM (Business Process Management).

\section{Systemic Model of Organizations}

From here on, the word organization will be used to refer to the complete company or any of its parts, since the company is made up of a number of organizations and each of these, in turn, is made up of other organizations. This deployment of the company, in organizations integrated by other organizations, must be done a limited number of times, until reaching organizations that still have a clear generic function and results that are relevant. That decision will have to be made by the person doing the analysis and sometimes, it may be necessary to reach a level of detail that will be inadequate and will have to return to some previous point of deployment.

An organization is, in general terms, a set of people who are dedicated to a generic activity necessary for the systems of other organizations to function and mainly the systems that generate the value that the company requires to exist. To develop the model that describes an organization based on systems, it is convenient to analyze the entire company as the organization under study. If we take this approach, the organization will have as expected results certain products that have value for customers and on which the existence of the company and all its internal organizations depends.

Every product is the result of a process that generates it and this constitutes the first axiom of the Specific Theory of Systems (STS), which is the name that seems appropriate for the set of knowledge that is generated from the definition of the system that was given at the beginning of this article. The above can be il- 
lustrated as follows (Figure 2).

If the product is a material good, then there must be inputs that feed the process, so that the actions carried out in it, transform them into the final product. In such a case, the situation can be illustrated with a diagram like the one shown in Figure 3.

It is necessary to clarify that in Figure 3, all the feeding processes are external to the main process and all of them generate materials as results. There cannot be any among these processes that only generate actions because if they are actions required by the main process, then that process must be a component and be part of the main system. It should be emphasized that the process is part of a system whose components can be observed and that in this case we are talking about the process that is made up of actions that happen and disappear, that is one of the problems that surround the processes, nobody is capable of Observe them as a whole, human beings can only observe one or two actions at most and since they are fleeting, we will see them and the next moment they will no longer be within reach of our observation.

From Figure 3 it is directly concluded that each of the feeding processes must have its own feeding processes, since the processes are only made up of actions generated by the components of the system and for it to be able to generate materials it has to receive inputs, so that each of the feeding processes has a diagram similar to that of Figure 1. If this is represented in a diagram, Figure 4 is obtained.

Based on the reasoning in the previous paragraph, the feeder processes that are drawn smaller, also have their feeder processes and theirs, so that theoretically successive levels of display can be obtained indefinitely. However,

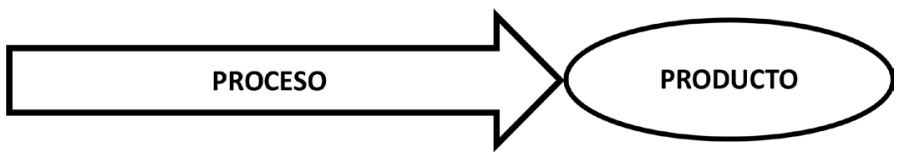

Figure 2. Process and product.

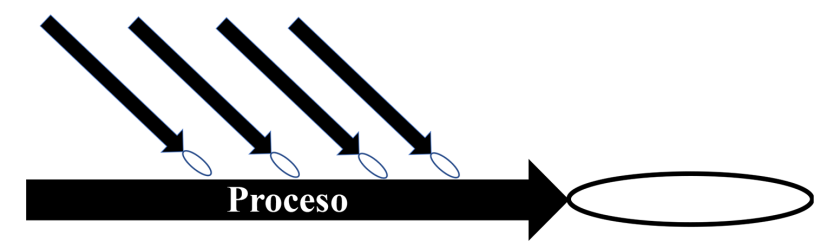

Figure 3. The system and its inputs.

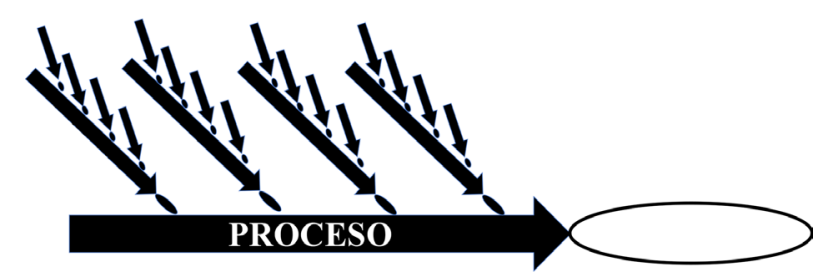

Figure 4. Second level systems. 
deployment ends when the inputs correspond to a process that belongs to another organization other than the one being analyzed or when the detail of the analysis that is carried out no longer requires further deployment.

For each product of the organization a network of systems is obtained with the structure shown in the previous figures, each process represents the existence of a system. It is necessary to clarify that, for example, Figure 3 is not a system of systems, since although each of the processes is part of a system, by definition the components of a system always and only generate actions and the processes of Figure 3, generate materials and cannot be considered components of a system. Therefore, we will call an entity like the one represented in Figure 3: a system network. Of course, Figure 4 is a set of system networks, which we will also call systems network, but it should be clear that systems networks are not systems. However, the components of systems are systems and therefore every system is a system of systems. This is the correct way to interpret the principle of recursion of the systems that the TGS postulates. This law is also complied with in systems networks, since by definition, a system network is made up of systems networks.

Then each product of an organization is associated with a network of systems that contains all the systems that are required to generate it. The organization will have as many system networks as there are products it generates, and the complexity of these networks will depend on the complexity of the product. The complexity may be greater because in reality, several or many of the network systems may be related to other systems in a network or to several networks, or to networks of other organizations, thus forming complex structures of relationships and effects.

An organization is made up of a set of organizations and each one of these functions based on one or more networks of systems, which can be related through structures that can cover conditions of little or high complexity. The systemic model of an organization is built with networks of systems that transform inputs from outside of it into specialized inputs required by the process that generates the final product. Final products are those that the organization supplies to other internal organizations as inputs or to customers in the event that the products are those that the company produces to satisfy its markets. Every organization has internal products that are required by other organizations within it and final products that are delivered to customers external to it. The company as a whole and each of the organizations that comprise it can be analyzed based on this systemic approach.

There is also the possibility that the organization is composed only of systems that do not generate material results and this makes it a system of systems. In the simplest case, the systems that make up the organization work in sequence, so that one begins its operation when its predecessor in the sequence finishes doing so, in this case, the systemic model is that of a line of systems that work in a coordinated manner. Each system at the end of the last action of its process has 
generated a change or event, therefore this systemic structure can be called a sequence of events. It is convenient to clarify that there is a sequence only in the case in which a system requires as an unavoidable antecedent that the event of the system that precedes it be concluded. If two systems are not sequential, then their results are independent and can be executed in any desired or convenient order. If they develop one after the other, they would be consecutive, but not sequential. If there is no sequence, the systems can operate in parallel or consecutively or in any time combination that is decided. This crucial difference between sequence and consecutiveness must always be kept in mind.

Systems networks always work in sequence since systems always supply a material input to the system that follows in sequence and as the material input is a requirement for the receiving system to act, then it is essential that the sequential order be maintained. between the supplying system and the receiving system. Of course, as illustrated in Figure 2, several different systems can be in sequence with the same receiver and the providers do not have to be in sequence, since there is no relationship between them. Of course, the operation of the receiving system sets them an order of execution so that the necessary actions are carried out sequentially on the inputs in order to generate the product.

In summary, an organization is made up of networks of systems or by systems of systems or by a combination of both structures, to develop a diagram that represents the systemic model of the organization that is to be described, arrows are used that represent the processes and they are placed in an appropriate way to describe the relationships that exist between them, when appropriate, other arrows will be used as connectors to describe the more complex relationships between processes. Although the diagram is representing processes, it should not be forgotten that every process represents a system of which it is a part, so when representing processes, systems are being represented.

From Figures 1-3, it is intuited that the systems model of an organization is built from its products, because once these have been identified, it is feasible to identify the processes that generate them and the inputs that are required and therefore the processes that generate them and thus you can continue as far as you want or until you find an input that comes from outside the organization.

It is very important to recognize that the systemic structure is unique as long as the products are the same, the processes can be improved but the relationships that exist between them will not change. The systemic structure does not depend on the organization chart or model that is adopted either. Commonly, companies work on the basis of organization charts developed with criteria for the division of tasks or with methodologies that seek some type of commercial strategy or customer or market service. For this reason, the components of the systems tend to be distributed in an illogical way in the functions determined in the organization, which leads to a malfunction of the systems and of the systems networks, since their different components obey to approaches, priorities and different interests with which they lose their coordination and coherence. The 
organization, therefore, should be designed based on the systemic structures that the products require, only in this way, will it be possible to obtain a true organization and management by processes. All other organizational designs, such as those designed to operate horizontal systems, for example, are utopian and inefficient from a systemic point of view.

Up to this point, the systemic structure that encompasses the systems that are dedicated to generating the products that allow an organization to create value for the people, processes or organizations that need them and act as their customers have been described. Figure 4 illustrates the systemic network that requires the generation of a product, but only includes all those systems that provide some material result for its generation and that therefore directly influence its quality. It must always be taken into account that every input that becomes part of a product affects it directly, precisely and definitively, if the input has a defect, it causes a defect in the final product.

In every organization there are systems that do not provide any input for the final products, but provide, for the most part, services that the productive systemic networks require to function. The most obvious services are, for example: lighting, buildings, furniture, cleaning, security, transportation, food, government procedures and many more, all these services are necessary for the productive systemic networks to function, but they have no influence on the products, since nothing that these services provide is incorporated as elements of the final products, in other words they do not provide any input to the products and therefore do not affect their quality in any way.

These services are generated by systems that are part of the organization, but that has not been considered in the productive systemic networks described above. Because they are of a different nature, they must be integrated into a different type of systemic networks or systems, these systems are called support systems and although they do not contribute with inputs, they are decisive for the functioning of productive systemic networks and all must exist and work for these networks to work. The support systems must have as a fundamental purpose, to contribute so that the productive systemic networks work perfectly.

Given its compulsory and unavoidable vocation of being at the service of productive systemic networks, its graphic representation must be made with a diagram like the one below, in which the orientation of the white arrows towards the final process is intended to express that vocation.

Figure 5 is the complete graphic representation of the systemic model that correctly describes how the systems that generate a product are integrated, we will call this representation, from here on: Structural Map of a product, although we can also say that it is the Structural Map of the process that generates the product, both interpretations are correct, since every product has only one process that generates it.

It should be noted that support systems do not generate material products and therefore, a set of support systems can be considered a system, therefore support 
systems can form a system of support systems. Support systems normally perform actions as final results and are therefore considered services, since a service is a set of actions. However, support systems can generate, as a result of their actions, certain enduring products that can be observed, analyzed and used at different times and by different users and in these cases, it can be considered that these systems provide their clients with tangible products., even if they are not materials to be used as inputs. Tangible products can be: a digital file or a document and that makes the support systems not only result in actions.

On the other hand, the support systems also have their feeding systems that provide them with services or tangible results and require, also support systems, therefore, the systemic model of an organization is built from the productive systemic networks and their systems. of support systems. Figure 6 illustrates the systemic model of an organization up to the second level of deployment. Most of the support systems are general and are repeated in all the Structural Maps of all the systems, but some systems may require specific support systems.

The systems that make up an organization can be social systems, in which the components are only people, but the common thing is that they are sociotechnical systems in which the components are people and machines that can be handled as tools or machines that perform complex actions of chemical, electrochemical, physicochemical, nuclear or any other type of reaction that cannot be replaced by human actions. However, the system formed by people and technological instruments is one and not two, as was considered in the Tavistoc Institute research. The sociotechnical system has human components and technological components, but they all make up a single system. This is applicable to both productive systemic networks and support systems.

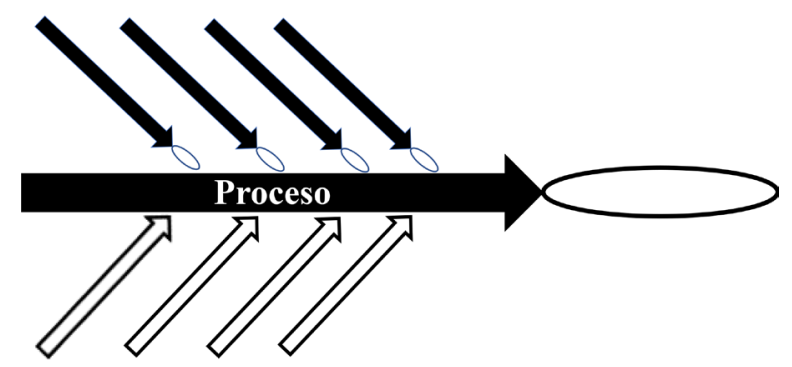

Figure 5. Structural map of a product.

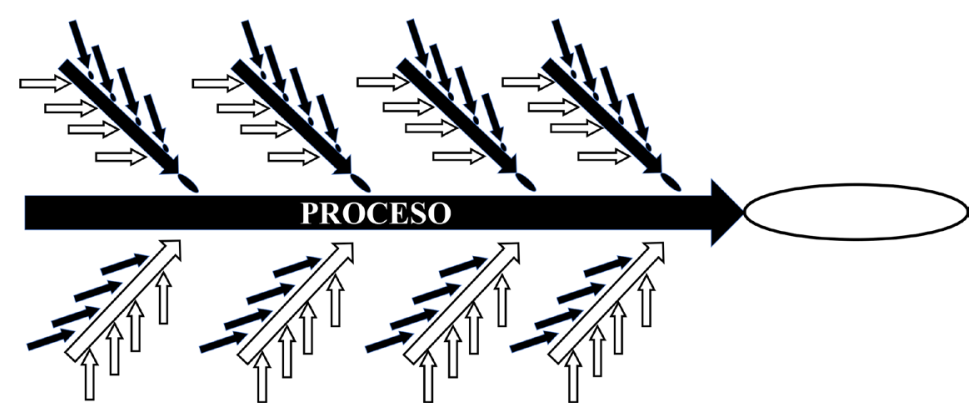

Figure 6. Complete structural map of a product. 


\section{Conclusion}

From a new definition of systems, it is possible to develop a new theory about systems, which has been called Specific Theory of Systems, which replaces the General Theory of Systems and provides a more solid and clear conceptual and theoretical basis applicable only to those entities that are true systems according to the aforementioned definition.

From the definition proposed by Pérez (2020c), a model can be deduced and theoretically supported that allows an organization to be described based on the systems that comprise it and allow it to function. This model is developed from the products or results that the organization generates and therefore the proposed systemic structures are the fundamental elements to ensure that the organization generates value and manages to remain current and productive.

The system model deduced based on the STS, is fully supported by this theory, however, it is necessary to carry out investigations that allow to confirm in reality that this model adequately represents what happens in a real organization, that is, to empirically verify that Systems Networks are the configuration on which organizations operate, regardless of their specialty and that the Structural Maps represent them adequately.

It is convenient to clarify that the people who work in an organization will surely not be aware of the existence of systems and systemic networks, but in spite of this they will exist and will be the basis of their operation and the structure on which the results are generated. The researcher's job will be to bring out the systems and their structures from the knowledge that the people who are part of them have.

This work was developed solely to deduce a hypothesis from the fundamental concepts of a theory, therefore it is not possible to conclude anything in relation to the validity of said model. In order to continue the investigation, it will be necessary to design a new empirical investigation that allows to contrast the hypothesis proposed here, with the reality that exists in organizations. This can be done through analytical studies carried out with groups of people who belong to specific organizations and with which it is possible to identify the systems that generate the final results of the organization and the supply systems of inputs and general services and develop one or two levels of detail, with which the hypothetical model proposed here could be verified or discarded. An attempt could also be made to develop a quantitative investigation based on the proposed model, which would require developing an instrument that would allow the latent variables to be measured and confirm the proposed hypothetical model.

\section{Conflicts of Interest}

The author declares no conflicts of interest regarding the publication of this paper. 


\section{References}

Adams, K., et al. (2014). Systems Theory as the Foundation for Understanding Systems. Systems Engineering, 17, 112-123. https://doi.org/10.1002/sys.21255

Benedict, T., et.al. (2013). BPM Common Body of Kwnowledge. USA: Association of Business Process Management Professionals.

Bertalanffy, L. (1950). The Theory of Open Systems in Physics and Biology. Sience, 111, 23-29. https://doi.org/10.1126/science.111.2872.23

Bertalanffy, L. (1968). Teoría General de los Sistemas. Cidad de México: Fondo de Cultura Económica.

Feigenbaum, A. (1951). Quality Control: Principles, Practice and Administration. 2 Pennsylvania Plaza, New York City: McGraw Hill Book Company Inc.

Ishikawa, K. (1986). Qué es el control total calidad: La modalidad japonesa. Editorial Norma.

Katz, D., \& Kahn, R. (1966). The Social Psychology of Organizations. Hoboken: John Wiley and Sons Inc.

Ostroff, F. (1999). The Horizontal Organization: What the Organization of the Future Actually Looks like and How It Delivers Value to Customers. Oxford: Oxford University Press.

Pérez, G. (2020a). On the Essence of Systems in General and Social Systems. Technium Social Sciences Journal, 628-642.

https://www.techniumscience.com/index.php/socialsciences/article/view/636/307

Perez, G. (2020b). On the Essence and Ontology of Systems. Open Science Journal, 5, 1-15. http://www.ajssmt.com/Papers/25125134.pdf https://doi.org/10.23954/osj.v5i3.2510

Pérez, G. (2020c). In the Search for a Precise Objective and True Definition of Systems. Asian Journal of Social Science and Management Technology, 2, 125-134. http://www.ajssmt.com/Papers/25125134.pdf

Rummler, G., \& Brache, A. (2013). Improving Performance: How to Manage the White Space on the Organization Chart. San Francisco, USA: John Wiley \& Sons, Inc.

Shewhart, W. (1930). Economic Quality Control of Manufactured Product. The Bell System Technical Journal, IX, 364-389. https://doi.org/10.1002/j.1538-7305.1930.tb00373.x

Trist, E. (1981). The Evolution of Socio-Thecnical Systems. Toronto, Ontario: Ontario Ministry of Labour, Ontario Quality of Working Life Centre. 